\title{
Light welding nanoparticles: from metal colloids to free-standing conductive metallic nanoparticle film
}

\author{
Fei Chen ${ }^{1}$, Shuzhen Yang ${ }^{2}$, Zhenjun $\mathrm{Wu}^{1}$, Wei $\mathrm{Hu}^{2 *}$, Jiawen $\mathrm{Hu}^{1 *}$ and Xiangfeng Duan ${ }^{1,3^{*}}$
}

\begin{abstract}
Bottom-up assembly of nanostructured thin films could offer an alternative low-cost approach to electronic thin films. However, such solution-processed thin films are often plagued by excessive inter-particle resistance and only exhibit limited current delivering capability. Here, we report a novel approach to fabricate highly conductive free-standing metallic thin film, accomplished by combining interfacial self-assembly of nanoparticles (NPs) and a light welding process. We found that light from a xenon lamp can weld adjacent $\mathrm{Ag}$ and $\mathrm{Au}$ NPs assembled at the water-air interface, forming a highly interconnected, free-standing metallic thin film structure with excellent electrical transport properties. With such a unique structure, the resultant thin metallic films show not only high flexibility and robustness, but also high conductivity comparable to bulk metallic thin films. Our studies offer a low-cost, room-temperature, and solution-processable approach to highly conductive metallic films. It can significantly impact solution-processable electronic and optoelectronic devices.
\end{abstract}

Keywords: interfacial self-assembly, nanoparticle, light welding, conductive metallic film, photodetector

\section{INTRODUCTION}

Metallic thin films have widespread applications, ranging from conductive and/or transparent electrode to chemical sensing platform [1]. Most metallic films are prepared by costly vacuum-based deposition processes, such as chemical vapor deposition $[2,3]$, thermal or electron beam evaporation [4], and molecular beam epitaxy [5], which usually require costly and sophisticated equipment with limited throughput.

Alternatively, solution-based processes may offer a sim- pler and lower-cost pathway for large scale processing of metallic thin films. For example, electric circuits can be printed using metallic nanoparticles (NPs) as "electric ink" [6,7]. Self-assembly of NPs at a fluid interface is another attractive method [1,8-12]. Because interfacial self-assembly of NPs usually produces a two-dimensional (2D) monolayer film, metallic thin films with well controlled thickness can be easily fabricated by choosing NPs with a desired diameter and by repeating the monolayers of the $2 \mathrm{D}$ film transferred onto the host substrate. Moreover, the properties of the metallic films can be further tuned by changing the particle shape, size, composition, and spacing. With these unique characteristics, the assembled metallic thin films can be used for catalysis [13,14], surface-enhanced Raman scattering substrate [15,16], and mirror [17]. Recently, NP monolayer film, assembled at the liquid-gas interface within the confined space at micron scale, has been constructed as strain gauge, which shows superior sensitivity and dynamic response to the externally acoustic vibration [18]. Therefore, interfacial self-assembly is also a powerful bottom-up strategy to make functional unit of the nanoscale devices from NPs [19]. However, such solution-assembled NP thin films are often plagued by excessive inter-particle resistance that limits current delivering capability $[20,21]$, which has severely limited their application as conductive electrodes in advanced electronic and optoelectronic devices.

To impart excellent charge transport properties to the NP thin films plagued by abundant inter-particle junctions, welding becomes technically imperative to reduce the resistance at the junctions. Recently, a number of studies have shown that cold-welding [22], lateral self-assembly

\footnotetext{
${ }^{1}$ State Key Laboratory for Chemo/Biosensing and Chemometrics, College of Chemistry and Chemical Engineering, Hunan University, Changsha 410082, China

${ }^{2}$ School of Physics and Electronics, Hunan University, Changsha 410082, China

${ }^{3}$ Department of Chemistry and Biochemistry, University of California, Los Angeles, California 90095, USA

*Corresponding authors (emails: jwhu@hnu.edu.cn (Hu J); huwei@hnu.edu.cn (Hu W); xduan@chem.ucla.edu (Duan X))
} 
[23], overgrowth of assembled NP film [24], and self-limited plasmonic welding [25] can effectively fill the interparticle junctions, making highly interconnected NP thin films with improved conductivity. These methods, however, are time-consuming, or need high-temperature annealing, which may cause severe damages to the host substrate, e.g., flexible polyethylene terephthalate (PET) film. Moreover, the electric properties of such films and their potential applications have not been fully studied.

Herein, we report a room temperature, solution-based approach to fabricate highly conductive metallic thin film by combining interfacial self-assembly of NPs and a light welding process. With a highly interconnected 2D network structure, the welded metallic NP thin film exhibits not only high flexibility and robustness but also high conductivity, making it an ideal low-cost electrode for the construction of electronic and optoelectronic devices (e.g., perovskite photodetectors).

\section{EXPERIMENTAL SECTION}

\section{Materials}

Silver nitrate, chloroauric acid, trisodium citrate, cyclohexane and ethanol were purchased from Sinopharm Chemical Reagent Co. Ltd (Shanghai, China). Lead(II) iodide and methylammonium iodide $\left(\mathrm{CH}_{3} \mathrm{NH}_{3} \mathrm{I}\right)$ were purchased from Xi'an Polymer Light Technology Corporation (China). All reagents are of analytical grade and were used without further purification. Before use, $\mathrm{CH}_{3} \mathrm{NH}_{3} \mathrm{I}$ was dissolved in isopropanol $\left(15 \mathrm{mg} \mathrm{mL}^{-1}\right)$ and all glasswares and stir bars were cleaned with aqua regia $(3: 1$ of $\mathrm{HCl}(37$ wt.\%) and $\mathrm{HNO}_{3}$ (65 wt.\%), v/v) solution (CAUTION: aqua regia solution is strongly corrosive and should be used with extreme care) and then rinsed thoroughly with deionized water.

\section{Interfacial self-assembly of NPs and light welding}

Ag NPs (60 nm) [26] and Au NPs with different sizes (16, 30,55 , and $70 \mathrm{~nm}$ ) [27] were synthesized by reduction of $\mathrm{AgNO}_{3}$ and $\mathrm{HAuCl}_{4}$ with citrate, respectively. After their synthesis, cyclohexane $(5 \mathrm{~mL})$ was gently covered on the surface of the metal colloids $(20 \mathrm{~mL})$ to produce a sharp water-oil interface. Upon quick addition of ethanol $(1.5 \mathrm{~mL})$, the metallic NPs in the colloids were induced to the wateroil interface, where they were trapped and spontaneously self-assembled into an NP thin film with metallic luster $[9,28]$. After careful removal of the oil using a syringe, the metallic NP thin film was left at the water-oil interface. Subsequently, the NP film was welded by light irradiation from a $50 \mathrm{~W}$ CEL-HXF 300 xenon lamp (CEAULICHT Co.,
Beijing, China). Depending on downstream characterizations and applications, the metallic NP thin film was transferred on a stainless steel mesh (for welded film only), Si wafer, PET film, or glass slide, which served as the host substrate.

\section{Construction of perovskite photodetector}

The welded metallic NP thin films were used as electrodes for the construction of perovskite photodetectors. Briefly, a glass slide $(15 \mathrm{~mm} \times 15 \mathrm{~mm} \times 1 \mathrm{~mm})$ was sequentially cleaned by sonication in acetone, ethanol, and deionized water (each for $15 \mathrm{~min}$ ). After that, a rectangular area (5 $\mathrm{mm} \times 15 \mathrm{~mm}$ ) was defined on the slide using a $3 \mathrm{M}$ tape, followed by transferring the welded metallic NP thin film. Tearing off the tape left a rectangular NP film on the substrate. Interdigital electrodes were made by scratching rectangular channels on the metallic NP film using a needle tip. After that, a thin $\mathrm{PbI}_{2}$ film $(60 \mathrm{~nm})$ was thermally evaporated onto the interdigital electrodes. Then, isopropanol solution of $\mathrm{CH}_{3} \mathrm{NH}_{3} \mathrm{I}\left(20 \mu \mathrm{L}, 15 \mathrm{mg} \mathrm{mL}^{-1}\right)$ was dropped on the $\mathrm{PbI}_{2}$ film, spinned at $3000 \mathrm{rpm}$ for $30 \mathrm{~s}$, and naturally dried. Finally, the glass slide was annealed on a hot-plate at $100^{\circ} \mathrm{C}$ for $20 \mathrm{~min}$, creating a uniform, dense perovskite $\left(\mathrm{CH}_{3} \mathrm{NH}_{3} \mathrm{PbI}_{3}\right)$ film ( $120 \mathrm{~nm}$ thickness) over the entire surface.

\section{Characterizations}

Scanning electron microscopy (SEM) and transmission electron microscopy (TEM) characterizations were conducted on a LIGMA microscope (Zeiss, Germany) and a JEM-2100F microscope (JEOL, Japan), respectively. For these characterizations, the samples were prepared by carefully transferring the metallic NP film at the water-air interface onto a carbon-coated $\mathrm{Cu}$ grid and a $\mathrm{Si}$ wafer, respectively. Electrochemical impedance spectroscopy (EIS) spectra were measured on an Autolab 302N electrochemical workstation using a two-electrode mode. For EIS measurements, the metallic NP film was transferred onto the gap (width: $2 \mathrm{~mm}$, length: $6 \mathrm{~mm}$ ) of a pair of $\mathrm{Cr} / \mathrm{Au}$ electrodes defined on a glass slide. During EIS measurements, the $I-V$ curve and Nyquist plot give the film's $I-V$ response and resistance, respectively. From the resistance of the film, its sheet resistance can be calculated by knowing its dimensions (the thickness of the film is nearly equivalent to the diameter of the NPs used for the assembly). The performance of the perovskite photodetector was determined on a Keithley 4200 semiconductor parameter analyzer (Keithley, United States) in atmosphere. To this end, a xenon lamp (PL-SPS1000, Perfect 
Light Co. Ltd., China) was used as monochromatic light source. Before measurement, the power density of the incident light was calibrated using a standard silicon photodetector (PM100D, Thorlabs, Germany). Multiplying the power density with the active area between the interdigital electrodes allowed the calculation of the irradiation power used. All the measurements were performed at room temperature.

\section{RESULTS AND DISCUSSION}

Fig. 1a shows the schematic illustration of the fabrication process to obtain the conductive NP thin films. By quickly adding ethanol into the biphasic mixtures of metallic colloids in water and cyclohexane, the metallic NPs quickly accumulates at the water-oil interface $[8,9]$. Once arriving at the interface, the NPs are irreversibly trapped therein, unable being detached by thermal activation from the Brownian motion. This is because the detaching energy caused by the interfacial tension is usually higher than the thermal energy by a few orders of magnitude $[28,29]$. This irreversible trapping constitutes the basis for the self-assembly of robust NP thin films at the fluid interface. The metallic NPs trapped therein spontaneously self-assemble into an NP thin film with metallic luster, indicating the formation of a densely packed film. After removal of the oil phase, the metallic NP thin film left at the water-air interface was welded by light irradiation using a $50 \mathrm{~W}$ xenon lamp. By monitoring the intensity attenuation of the plasmon peak, the percent of NPs transferred to the water-air interface can be evaluated. For example, addition of ethanol induces about $40 \%$ of the $55 \mathrm{~nm}$ Au NPs to the water-air interface, while light duration of $1 \mathrm{~h}$ can drive another $20 \%$ of NPs to the interface (Fig. S1, Supplementary information).

Fig. 1b shows the digital photograph of an Ag NP thin film welded under light irradiation for $1 \mathrm{~h}$. The total area of the film assembled is as large as $\sim 12 \mathrm{~cm}^{2}$. By properly controlling the concentration of NPs in the starting solution, the NP thin films can be assembled to cover the whole interface area. In this way, the thin films can be readily scaled-up by increasing the interface area and the quantity of NPs used for the assembly. Interestingly, the welded Ag NP thin film exhibits very high mechanical strength, and can be readily fished out by a stainless steel mesh to form a stable free-standing thin film supported by the mesh (Fig. 1c). With such a high flexibility and robustness, the welded thin films can be transferred onto diverse host substrate. For example, the films transferred on a flexible PET substrate clearly show metallic reflection, confirming the densely packed NP thin films. Additionally, such thin film is highly robust and does not show obvious cracks upon

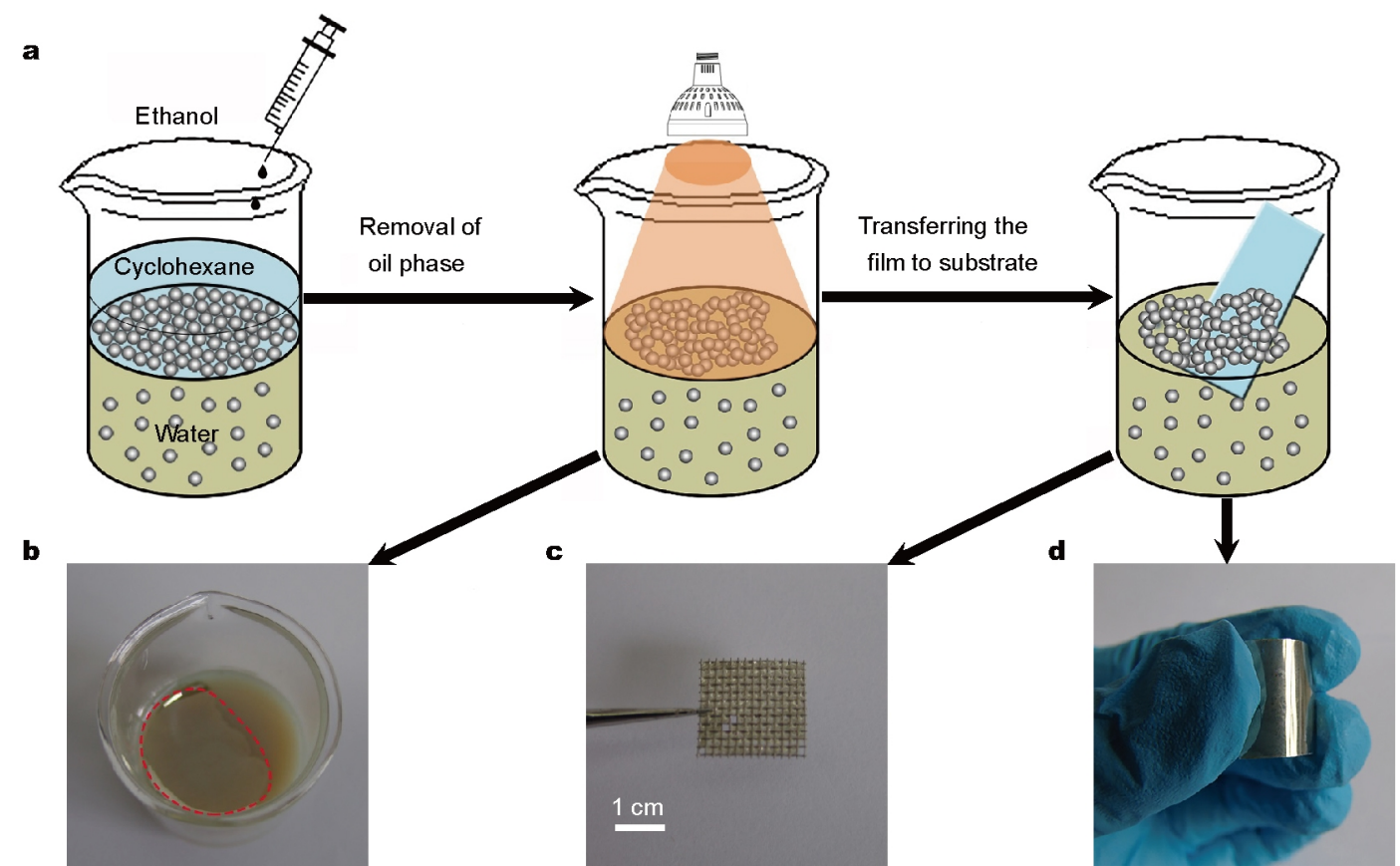

Figure 1 Fabrication and transferring of the NP thin films. (a) Schematic illustration of the fabrication process of the metallic NP film accomplished by combining interfacial self-assembly of NPs and a light welding process; (b, c, and d) optical images of the welded Ag NP thin films at the water-air interface, on a stainless steel mesh, and on a PET film. 

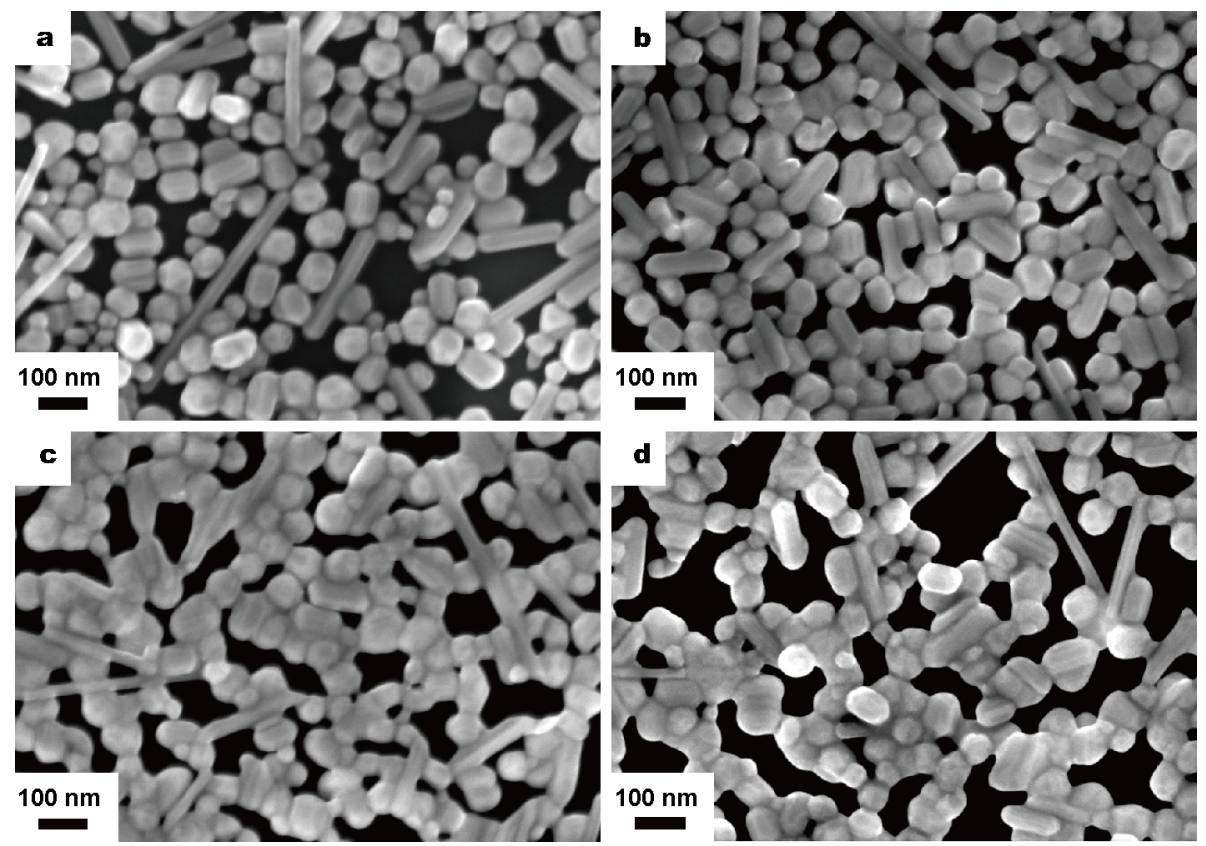

Figure 2 SEM images of the silver NP thin films welded by light irradiation at the water-air interface for 0 (a), 20 (b), 40 (c), and 60 min (d).

mechanical bending (Fig. 1d).

SEM and TEM studies were used to examine the welding extent of the NP film. Fig. 2 shows the SEM images for the Ag NP thin film welded by light irradiation for different duration. As observed previously, the NP thin film assembled at the water-air interface shows a typical monolayer structure dotted with small voids $[9,28,30]$. Before light welding, most NPs only physically contact with each other, with abundant inter-particle junctions in the range of 1-5 nm. With increasing welding time, it is apparent that the NPs in the film were gradually welded together, and finally fully merged together to form a highly interconnected $2 \mathrm{D}$ network structure. The diameter and shape of the filament in the network are nearly identical to their parent particles, implying that welding occurs only at particle-particle junctions.

To further evaluate whether our approach is general, we also applied the light welding approach to other metallic NPs (e.g., Au NPs). To this end, Au NPs were synthesized using a previously reported approach [27], and assembled at the water-oil interface using the same procedures described above to obtain Au NP thin films, which was then subjected to light irradiation for variable duration. The SEM studies of Au NP thin films clearly show that the initially discrete NPs can be welded into a highly interconnected network structure upon increasing the irradiation time (Fig. 3). The generality of light welding approach is also further demonstrated by welding Au NPs of different sizes (Fig. S2). The nature of particle-particle interface connection of the welded NPs can be more clearly revealed by TEM observation. TEM images clearly show that inter-particle junctions are completely fused together, for the Ag and Au NP thin films after light welding for $1 \mathrm{~h}$ (Fig. 4). These microscopic observations strongly indicate that light plays an essential role in welding adjacent $\mathrm{Ag}$ and $\mathrm{Au}$ NPs assembled at the water-air interface. In addition to light duration, its power density may also have an important impact on the welding. We thus examined the morphology of the NP films welded using variable light power density (Fig. S3). With increasing power density from 1.8 to $4.1 \mathrm{~W} \mathrm{~cm}^{-2}$, the connection extent becomes more apparent for the $55 \mathrm{~nm} \mathrm{Au} \mathrm{NP} \mathrm{film,} \mathrm{revealing} \mathrm{that} \mathrm{light} \mathrm{power}$ density also plays an important role in the welding. We further studied the light welding using other sources, such as $632.8 \mathrm{~nm}$ laser $\left(0.064 \mathrm{~W} \mathrm{~cm}^{-2}\right.$, expanded into a $2-\mathrm{cm}$ beam via a beam expander), tungsten-halogen lamp $(\sim 0.7$ $\left.\mathrm{W} \mathrm{cm}{ }^{-2}\right)$, and summer sunlight $\left(\sim 0.1 \mathrm{~W} \mathrm{~cm}^{-2}\right)$. However, their welding effect is negligible (Fig. S4) maybe because their power density is far lower than that of the xenon lamp used $\left(2.1 \mathrm{~W} \mathrm{~cm}^{-2}\right)$.

Light welding at the water-air interface can be largely attributed to the light-driven Ostwald ripening. In general, NPs can experience an Ostwald ripening process [31], in which solute atoms dynamically dissolve from NPs, and reattach to a location that is energetically comparable or more favorable than the original site, such as locations 


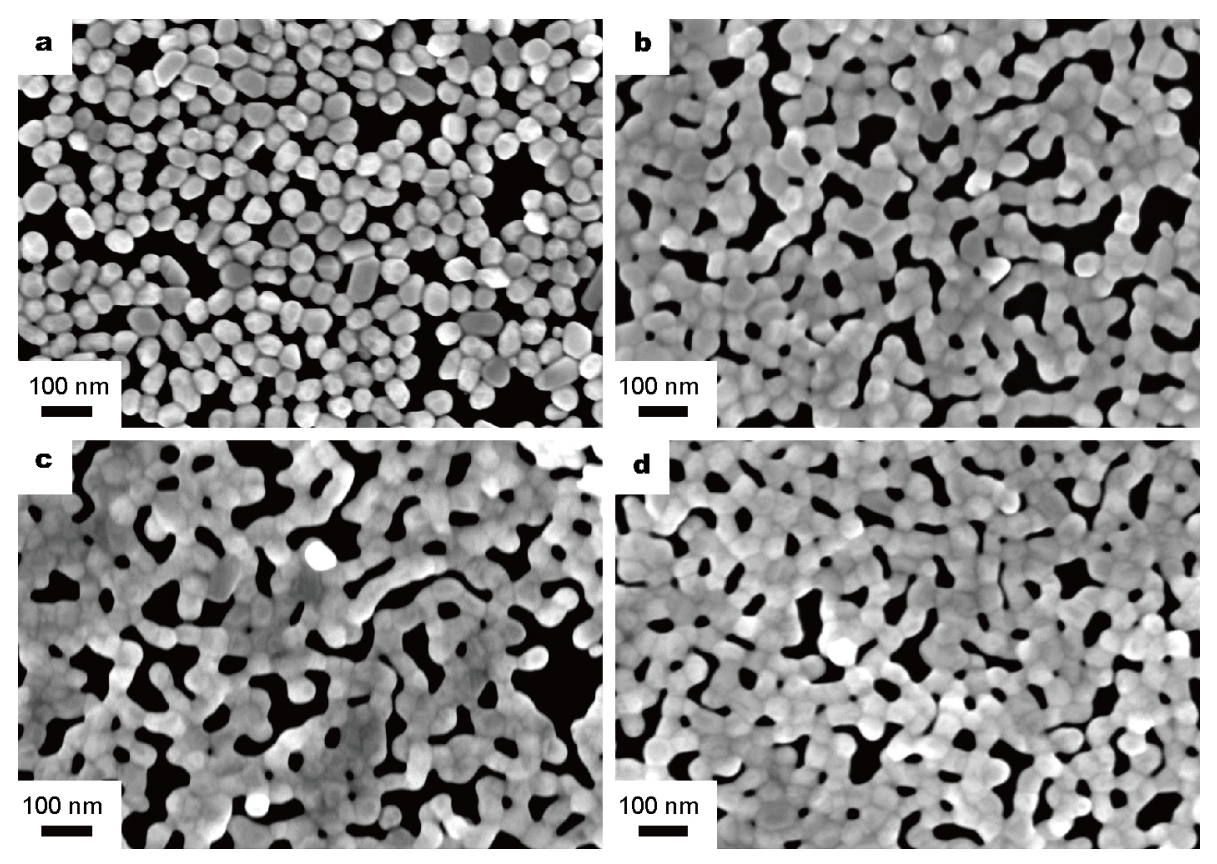

Figure 3 SEM images of the $55 \mathrm{~nm}$ Au NP thin films welded by light irradiation at the water-air interface for 0 (a), 20 (b), 40 (c), and $60 \mathrm{~min}$ (d).
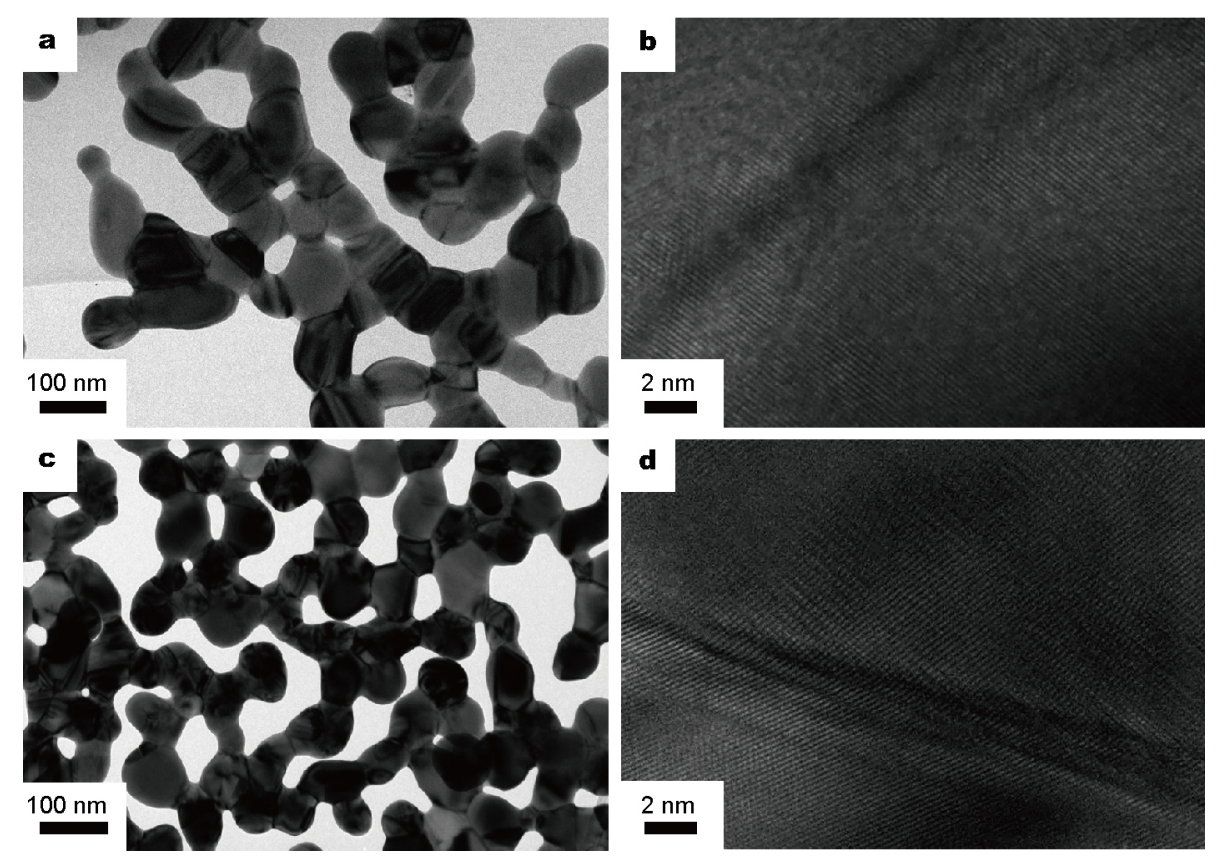

Figure 4 TEM images of the welded metallic NP thin films. (a and c) Normal TEM images for Ag and Au NP thin films welded by light irradiation at the water-air interface for $1 \mathrm{~h}$; (b and d) HRTEM images of the particle-particle junctions for the welded Ag and Au NP thin films shown in (a) and (c).

with smaller curvature or more coordinated neighbours. Consequently, the larger NPs grow at the expense of the smaller ones. The situation will change dramatically if the NPs are densely assembled at the water-air interface with close inter-particle distance. In such case, the energetically favorable sites are more likely located at the NP-NP interface with negative curvature, where the reattachment of dissolved $\mathrm{Ag}$ or $\mathrm{Au}$ atoms would lead to the formation of continuous network structure [22,23]. At room temperature, it, however, usually needs at least a few days to accomplish the whole welding process because Ostwald ripening is a slow thermodynamic process. Interestingly, 
we found that light can largely accelerate the welding of the densely packed film at the water-air interface (Figs 2-4). That is, light further activates the dissolution and rearrangement of $\mathrm{Ag}$ or $\mathrm{Au}$ atoms, thereby driving the Ostwald ripening and the welding process. The light-driven Ostwald ripening can be attributed to the unique localized surface plasmonic properties of Ag and Au NPs [32]. The surface plasmon resonance can create a very strong local field, especially at the NP-NP junctions [33], which can activate the dissolution and rearrangement of metallic atoms at the interface to fuse the NP into physically connected chains. In this case, the underlying solution offers an excellent medium for the dissolution, transport, and reattachment of the atoms. In contrast, the monolayer Ag NP thin film transferred on a Si host substrate cannot be welded together under the same light irradiation because it lacks solution pathway for the dissolution and movement of the metallic atoms (Fig. S5). In addition to light-driven Ostwald ripening, plasmonic heating may also contribute to the acceleration of the welding process. Plasmon can strongly absorb light and finally decay into local heat [34]. With this photo-thermal property, plasmonic materials have been exploited as photo-thermal agents to treat cancer [35] and plasmonic heaters to produce potable water $[36,37]$. Recent studies have also shown that plasmonic heating can melt crosslinked Ag nanowires at the junctions, but typically require much higher light intensity $\left(\sim 30\right.$ vs. $\left.\sim 2.1 \mathrm{~W} \mathrm{~cm}^{-2}\right)$ [25].

With a highly interconnected network structure after the light wedding process, the resultant metallic NP thin films exhibit excellent electron conductivity. Electron transport studies of the NP thin films show nearly perfect linear current-voltage $(I-V)$ behavior (Figs $5 \mathrm{a}$ and $\mathrm{b}$ ), indicating that the metallic NP films have only an Ohmic resistance and behave like pure resistors. The current increases continuously with increasing duration of light welding process. Taking the Ag NP thin film as an example, the as-assembled NP thin film shows a relatively high sheet resistance of $55.33 \Omega /$ sq, which rapidly decreases to $14.67 \Omega /$ sq after $5 \mathrm{~min}$ of light welding process, and further decreases with increasing welding time. Overall, the sheet resistance can be decreased by 30 times to reach $1.83 \Omega /$ sq after $1 \mathrm{~h}$ of light welding process (Fig. $5 \mathrm{c}$ ), clearly demonstrating the significant impact of light welding on the electronic properties of the Ag NP thin films. The same conclusion can be reached for the $55 \mathrm{~nm}$ Au NP thin films (Fig. 5d). Moreover, their sheet resistance, upon light welding for $15 \mathrm{~min}$, decreases from 12.4 to $2.1 \Omega$ /sq with increasing particle size from 16 to $70 \mathrm{~nm}$ (Fig. S6). This is because the welded NP film becomes thicker if the film is made of larger particles. Com-
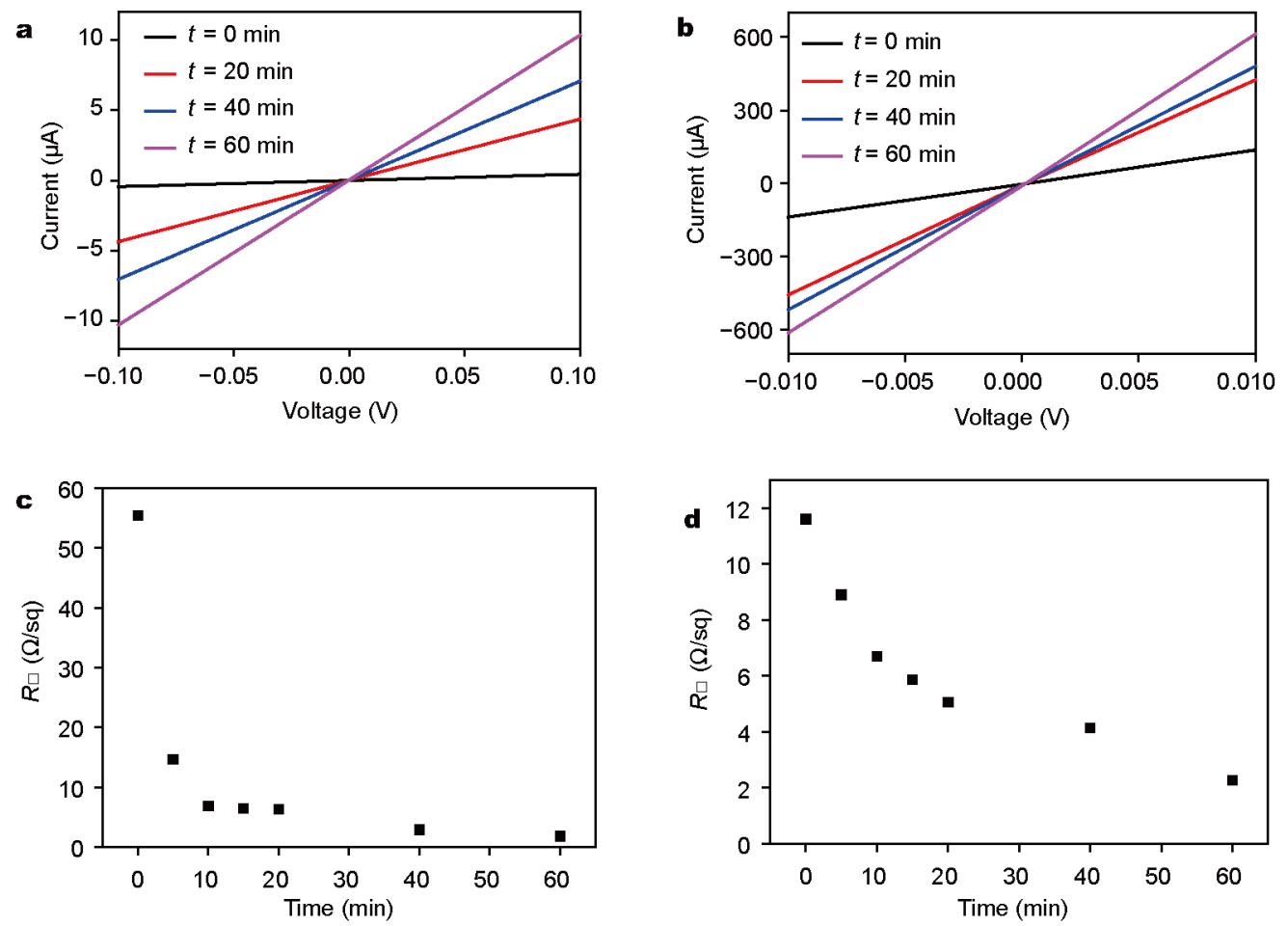

Figure 5 Electrical properties of the Ag and $55 \mathrm{~nm}$ Au NP thin films. (a and b) $I-V$ curves of the Ag and Au NP film welded by light irradiation for different duration; ( $\mathrm{c}$ and d) corresponding sheet resistances $\left(R_{\square}\right)$ for the films studied in (a) and (b). 
paring the sheet resistance of the welded NP thin film with that of bulk Ag and Au films of the same equivalent thickness (bulk Ag film: $0.28 \Omega /$ sq; bulk Au film: $0.44 \Omega / s q$ ) [38], our light-welded NP thin film shows highly comparable sheet resistance, highlighting that the light welding approach can produce highly interconnected network structure and offer efficient electron pathways.

Previous studies have suggested that 3D porous Au film has many unique physicochemical properties for diverse applications $[39,40]$. The same is true for our conductive $\mathrm{NP}$ thin films for the nature of 2D porous metallic thin film. In addition, the solution-assembled NP thin film may be readily as the conductive electrodes for functional electronic and optoelectronic devices. To this end, we used the welded Ag NP thin film as a part of the electric wire to light up an light-emitting diode (LED) device (Fig. 6a), implying the potential of such film as electrode in advanced electronic and optoelectronic devices. We further constructed photodetectors using the conductive Ag NP thin film as interdigital electrodes and $\mathrm{CH}_{3} \mathrm{NH}_{3} \mathrm{PbI}_{3}$ film as the active layer (Fig. 6b). The $I$ - $V$ curves of the devices show nearly linear characteristics, indicating the formation of a good a
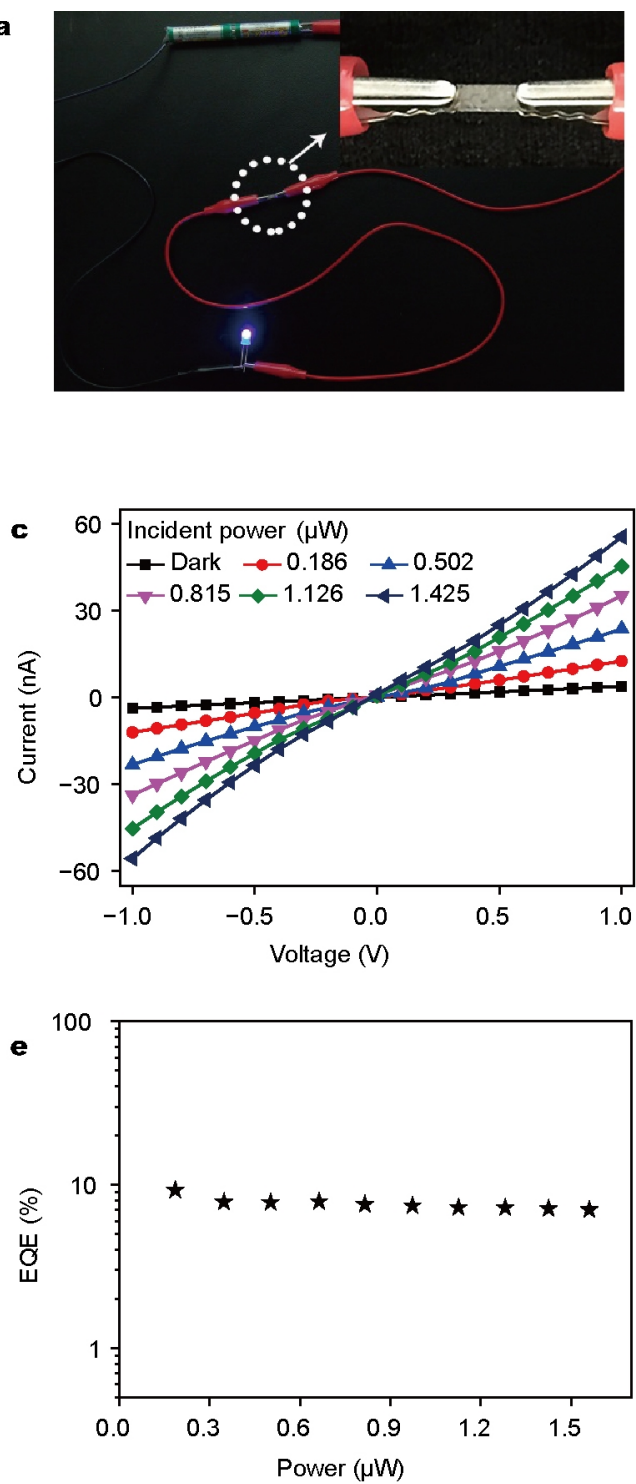

b

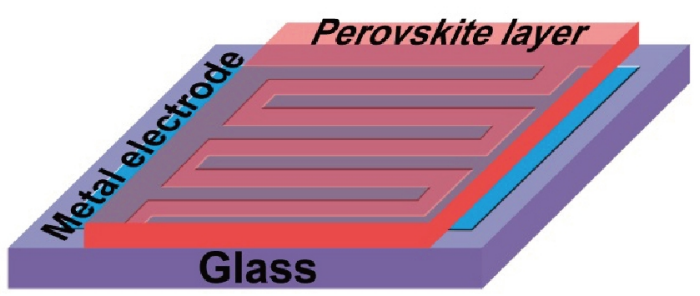

d
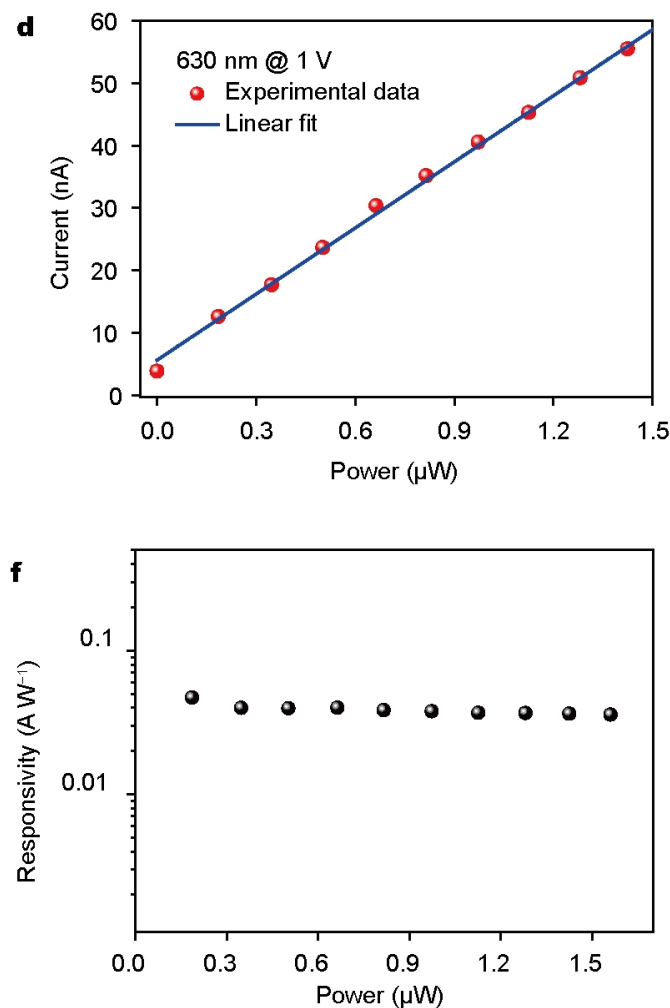

Figure 6 Performance of the hybrid perovskite photodetectors constructed from the conductive Ag NP films. (a) Photograph of a blue LED lightened using conductive Ag NP film as a part of the electric wiring; (b) schematic illustration of the photodetector; (c) $I$ - $V$ curves of a photodetector constructed from the conductive Ag NP thin film under $630 \mathrm{~nm}$ light irradiation; (d) light power-dependent photocurrent of the photodetector illuminated at 630 $\mathrm{nm}$ and at a bias voltage of $1.0 \mathrm{~V}$; EQE (e) and responsivity (f) of the photodetector illuminated by light irradiation at different powers. 
metal-semiconductor contact between the Ag NP film electrodes and the perovskite layer (Fig. 6c). Moreover, the photocurrents steadily increase with increasing irradiation power density and exhibit a good linear relationship with the irradiation power (Fig. 6d), suggesting that the photodetector can detect light irradiation over a wide range of powers. To further evaluate the performance of the constructed photodetector, we analyzed the external quantum efficiency $\left(\mathrm{EQE}=I_{\mathrm{ph}} / q \Phi\right)$ and responsivity $\left(R=I_{\mathrm{ph}} / P_{\mathrm{In}}\right)$ of the device, in which $I_{\mathrm{ph}}, q, \Phi$ and $P_{\mathrm{In}}$, are the photocurrent, elementary charge, the photon flux, and the power of incident light, respectively. EQE and $R$ are two critical parameters determining the performance of a photodetector because they represent the number of photo carriers produced per photon and the ratio of photocurrent to incident light intensity, respectively. Figs $6 e$ and $\mathrm{f}$ show, respectively, the EQE and $R$ of the photodetector achieved at bias voltage of $1 \mathrm{~V}$. Within irradiation power range of $0-1.6 \mu \mathrm{W}$, the achieved $R$ is about $0.05 \mathrm{~A} \mathrm{~W}^{-1}$ and EQE can generally reach up to $\sim 9 \%$, both of which are higher than that reported for ITO-contacted perovskite photodetectors $[41,42]$. These results indicate the photo-generated charge carriers in the perovskite layer can be effectively collected by the electrodes made of conductive Ag NP film. Thus, the combination of interfacial self-assembly of NPs and a light welding process offers a low-cost, room-temperature, and solution-processable avenue to fabricate highly conductive metallic thin electrodes for the use in advanced electronic and optoelectronic devices.

\section{CONCLUSIONS}

In conclusion, the combination of interfacial self-assembly of $\mathrm{Ag}$ and $\mathrm{Au}$ NPs and light welding of them at the water-air interface allows the fabrication of highly interconnected 2D NP thin films. The resultant metallic NP film not only has high flexibility and robustness, but also has a high conductivity, which is comparable to that of bulk $\mathrm{Ag}$ and Au films. Our studies define a low-cost, solution-processed, and room temperature-operated approach to fabricate high quality electrodes for functional electronic and optoelectronic devices. This will advance not only the applications of the NP thin films themselves (e.g., for the design of novel chemical sensors) but also the development of all solution-processed electronic and optoelectronic circuits.

Received 9 October 2016; accepted 2 November 2016; published online 7 December 2016

1 Liu JW, Liang HW, Yu SH. Macroscopic-scale assembled nanowire thin films and their functionalities. Chem Rev, 2012, 112:
4770-4799

2 Choy K. Chemical vapour deposition of coatings. Prog Mater Sci, 2003, 48: $57-170$

3 Rill MS, Plet C, Thiel M, et al. Photonic metamaterials by direct laser writing and silver chemical vapour deposition. Nat Mater, 2008, 7: 543-546

4 Cherkaoui K, Monaghan S, Negara MA, et al. Electrical, structural, and chemical properties of $\mathrm{HfO}_{2}$ films formed by electron beam evaporation. J Appl Phys, 2008, 104: 064113-064113

5 Harzer TP, Djaziri S, Raghavan R, et al. Nanostructure and mechanical behavior of metastable $\mathrm{Cu}-\mathrm{Cr}$ thin films grown by molecular beam epitaxy. Acta Mater, 2015, 83: 318-332

6 Kamyshny A, Magdassi S. Conductive nanomaterials for printed electronics. Small, 2014, 10: 3515-3535

7 Li Y, Wu Y, Ong BS. Facile synthesis of silver nanoparticles useful for fabrication of high-conductivity elements for printed electronics. J Am Chem Soc, 2005, 127: 3266-3267

8 Li YJ, Huang WJ, Sun SG. A universal approach for the self-assembly of hydrophilic nanoparticles into ordered monolayer films at a toluene/water interface. Angew Chem Int Ed, 2006, 45: 2537-2539

9 Reincke F, Hickey SG, Kegel WK, et al. Spontaneous assembly of a monolayer of charged gold nanocrystals at the water/oil interface. Angew Chem Int Ed, 2004, 43: 458-462

10 Guo Q, Xu M, Yuan Y, et al. Self-assembled large-scale monolayer of Au nanoparticles at the air/water interface used as a SERS substrate. Langmuir, 2016, 32: 4530-4537

11 Hu L, Chen M, Fang X, et al. Oil-water interfacial self-assembly: a novel strategy for nanofilm and nanodevice fabrication. Chem Soc Rev, 2012, 41: 1350-1362

12 Tao A, Sinsermsuksakul P, Yang P. Tunable plasmonic lattices of silver nanocrystals. Nat Nanotech, 2007, 2: 435-440

13 Xiao S, Xiao F, Hu Y, et al. Hierarchical nanoporous gold-platinum with heterogeneous interfaces for methanol electrooxidation. Sci Rep, 2014, 4: 4370

14 Duan MY, Liang R, Tian N, et al. Self-assembly of Au-Pt core-shell nanoparticles for effective enhancement of methanol electrooxidation. Electrochim Acta, 2013, 87: 432-437

15 Zhang L. Self-assembly Ag nanoparticle monolayer film as SERS substrate for pesticide detection. Appl Surface Sci, 2013, 270: 292-294

16 Cecchini MP, Turek VA, Paget J, et al. Self-assembled nanoparticle arrays for multiphase trace analyte detection. Nat Mater, 2012, 12: $165-171$

17 Fang PP, Chen S, Deng H, et al. Conductive gold nanoparticle mirrors at liquid/liquid interfaces. ACS Nano, 2013, 7: 9241-9248

18 Zhang C, Li J, Yang S, et al. Closely packed nanoparticle monolayer as a strain gauge fabricated by convective assembly at a confined angle. Nano Res, 2014, 7: 824-834

19 Yi L, Jiao W, Wu K, et al. Nanoparticle monolayer-based flexible strain gauge with ultrafast dynamic response for acoustic vibration detection. Nano Res, 2015, 8: 2978-2987

$20 \mathrm{Lu} \mathrm{H}$, Zhang D, Ren X, et al. Selective growth and integration of silver nanoparticles on silver nanowires at room conditions for transparent nano-network electrode. ACS Nano, 2014, 8: 10980-10987

21 Mutiso RM, Sherrott MC, Rathmell AR, et al. Integrating simulations and experiments to predict sheet resistance and optical transmittance in nanowire films for transparent conductors. ACS Nano, 2013, 7: 7654-7663

22 Liu C, Li YJ, Sun SG, et al. Room-temperature cold-welding of gold nanoparticles for enhancing the electrooxidation of carbon monoxide. Chem Commun, 2011, 47: 4481-4483

23 Wang MH, Li YJ, Xie ZX, et al. Fabrication of large-scale one- 
dimensional $\mathrm{Au}$ nanochain and nanowire networks by interfacial self-assembly. Mater Chem Phys, 2010, 119: 153-157

24 Xia H, Ran Y, Li H, et al. Freestanding monolayered nanoporous gold films with high electrocatalytic activity via interfacial self-assembly and overgrowth. J Mater Chem A, 2013, 1: 4678

25 Garnett EC, Cai W, Cha JJ, et al. Self-limited plasmonic welding of silver nanowire junctions. Nat Mater, 2012, 11: 241-249

26 Lee PC, Meisel D. Adsorption and surface-enhanced Raman of dyes on silver and gold sols. J Phys Chem, 1982, 86: 3391-3395

27 Frens G. Controlled nucleation for the regulation of the particle size in monodisperse gold suspensions. Nat Phys Sci, 1973, 241: 20-22

$28 \mathrm{Xu} \mathrm{L}, \mathrm{Han} \mathrm{G}, \mathrm{Hu} J$, et al. Hydrophobic coating- and surface active solvent-mediated self-assembly of charged gold and silver nanoparticles at water-air and water-oil interfaces. Phys Chem Chem Phys, 2009, 11: 6490-6497

29 Lin Y, Skaff H, Emrick T, et al. Nanoparticle assembly and transport at liquid-liquid interfaces. Science, 2003, 299: 226-229

30 Duan H, Wang D, Kurth DG, et al. Directing self-assembly of nanoparticles at water/oil interfaces. Angew Chem, 2004, 116 5757-5760

31 Voorhees PW. The theory of Ostwald ripening. J Stat Phys, 1985 38: 231-252

32 Camden JP, Dieringer JA, Zhao J, et al. Controlled plasmonic nanostructures for surface-enhanced spectroscopy and sensing. Acc Chem Res, 2008, 41: 1653-1661

33 Ding SY, Yi J, Li JF, et al. Nanostructure-based plasmon-enhanced Raman spectroscopy for surface analysis of materials. Nat Rev Mater, 2016, 1: 16021

34 Baffou G, Quidant R, Girard C. Heat generation in plasmonic nanostructures: influence of morphology. Appl Phys Lett, 2009, 94: 153109

35 Hirsch LR, Stafford RJ, Bankson JA, et al. Nanoshell-mediated near-infrared thermal therapy of tumors under magnetic resonance guidance. Proc Natl Acad Sci USA, 2003, 100: 13549-13554 nanoparticles for plasmon-enhanced solar desalination. Nat Photon, 2016, 10: 393-398

37 Wang Z, Liu Y, Tao P, et al. Bio-inspired evaporation through plasmonic film of nanoparticles at the air-water interface. Small, 2014, 10: 3234-3239

38 Vand V. A theory of the irreversible electrical resistance changes of metallic films evaporated in vacuum. Proc Phys Soc, 1943, 55: 222-246

39 Fujita T, Tokunaga T, Zhang L, et al. Atomic observation of catalysis-induced nanopore coarsening of nanoporous gold. Nano Lett, 2014, 14: 1172-1177

40 Li D, Zhu Y, Wang H, et al. Nanoporous gold as an active low temperature catalyst toward CO oxidation in hydrogen-rich stream. Sci Rep, 2013, 3: 3015

41 Dou L, Yang YM, You J, et al. Solution-processed hybrid perovskite photodetectors with high detectivity. Nat Commun, 2014, 5: 5404

42 Xia HR, Li J, Sun WT, et al. Organohalide lead perovskite based photodetectors with much enhanced performance. Chem Commun, 2014, 50: 13695-13697

Acknowledgments This work was supported by the National Natural Science Foundation of China (21673070 and 61528403), the Opened Fund of the Chinese State Key Laboratory on Integrated Optoelectronics (IOSKL2015KF29), and Hunan University.

Author contributions Chen F performed the main experiments and wrote the draft of this manuscript. Hu J, Duan X and $\mathrm{Hu}$ W directly guided and conducted this research including the design, modifying and polishing work related to this manuscript. Yang S and Wu Z provided help in the fabrication of devices and methods of characterization. All authors contributed to the general discussion about this work.

Conflict of interest The authors declare that they have no conflict of interest.

Supplementary information Supplementary data are available in the online version of the paper. 

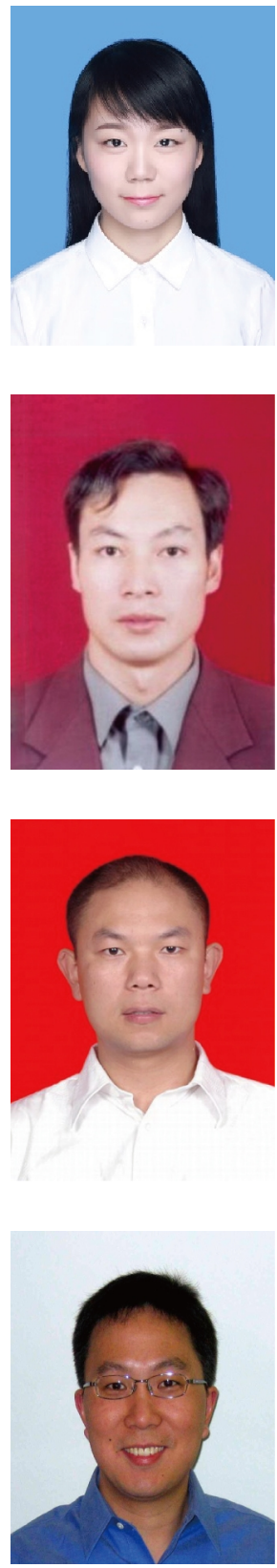

Fei Chen is a postgraduate master student of the College of Chemistry and Chemical Engineering, Hunan University. She joined Prof. Hu's group in 2014 and her current research interests include self-assembly of nanomaterials and nanoporous gold film for electrocatalysis.
Jiawen Hu is a professor of chemistry at the College of Chemistry and Chemical Engineering, Hunan University. He received his PhD degree in physical chemistry from Jilin University in 2002, followed by a period of postdoctoral research at Xiamen University. His research interests include surface-enhanced Raman scattering; synthesis, characterization, and self-assembly of nanomaterials; and capacitive desalination.
Wei Hu was born in 1979. He is a lecturer of Hunan University. He obtained his PhD degree in microelectronics and solid-state electronics from Jilin University in 2007. His research interests mainly focus on synthesis, characterization, and application of semiconducting nanomaterials for opto-electronic devices.
Xiangfeng Duan received the BSc degree in chemistry from the University of Science and Technology of China in 1997, and the MSc degree in chemistry and PhD degree in physical chemistry from Harvard University in 1999 and 2002, respectively. He is currently a professor at the Department of Chemistry and Biochemistry, University of California, Los Angeles. His research interests include nanoscale materials, devices and their applications in future electronics, energy science, and biomedical science.

\section{光焊接纳米粒子: 从金属溶胶到自支撑、导电性金属薄膜}

陈菲 ${ }^{1}$, 杨抒瑧 ${ }^{2}$, 吴振军 ${ }^{1}$, 胡伟 $^{2^{*}}$, 胡家文 ${ }^{1^{*}}, \mathbf{E}^{\text {镶锋 }}{ }^{1,3^{*}}$

摘要 金属薄膜在透明导电极、化学传感器、催化和光电器件等方面具有广泛应用. 发展溶液加工技术可以大幅度降低金属薄膜的制作 成本, 灵活地调控其性能, 从而促进其在多方面的应用. 我们发现氙灯光源可以高效地焊接在水-气界面上自组装的金和银纳米粒子薄膜; 其焊接程度取决于光照时间和光强度. 最终, 自组装的纳米粒子膜形成一种自支撑、高度交联的网状结构, 并具有和同样厚度的体相金属 薄膜相当的导电性. 这一发现将纳米粒子界面自组装技术与光焊接技术相结合, 可以将液相金属溶胶加工成高柔性、鲁棒性和导电性的 金属粒子薄膜. 这不仅可以促进金属粒子薄膜自身的应用, 而且可以促进全液相加工的电子和光电器件的发展. 例如, 利用该金属粒子薄膜 作为叉指电极的钻铁矿光电探测器展现出了良好的性能. 